\title{
Gradhiva
}

GRADHIV

Revue d'anthropologie et d'histoire des arts

$31 \mid 2020$

L'idéal du musicien et l'âpreté du monde

\section{Avec Frédéric Ramel. Un musicien peut-il adoucir les relations internationales?}

Denis Laborde et Frédéric Ramel

\section{(2) OpenEdition}

1 Journals

Édition électronique

URL : http://journals.openedition.org/gradhiva/5106

DOI : 10.4000/gradhiva.5106

ISSN : 1760-849X

Éditeur

Musée du quai Branly Jacques Chirac

Édition imprimée

Date de publication : 2 septembre 2020

Pagination : 113-127

ISBN : 978-2-35744-131-6

ISSN : 0764-8928

Référence électronique

Denis Laborde et Frédéric Ramel, « Avec Frédéric Ramel. Un musicien peut-il adoucir les relations internationales ? », Gradhiva [En ligne], 31 | 2020, mis en ligne le 31 mars 2021, consulté le 02 avril 2021. URL : http://journals.openedition.org/gradhiva/5106 ; DOI : https://doi.org/10.4000/gradhiva. 5106

(c) musée du quai Branly 


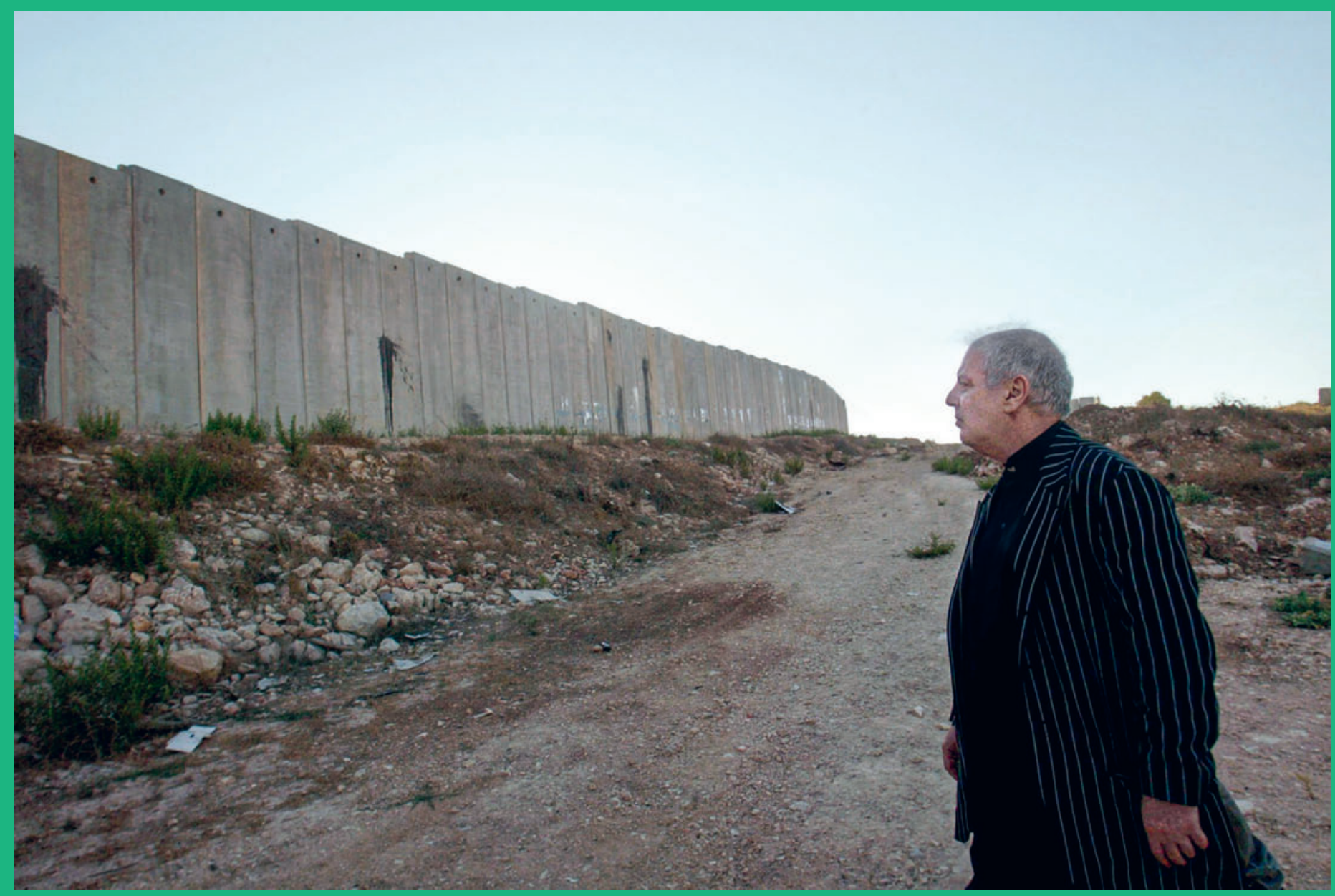


Avec

Frédéric Ramel.

Un musicien

peut-il adoucir

les relations

internationales?

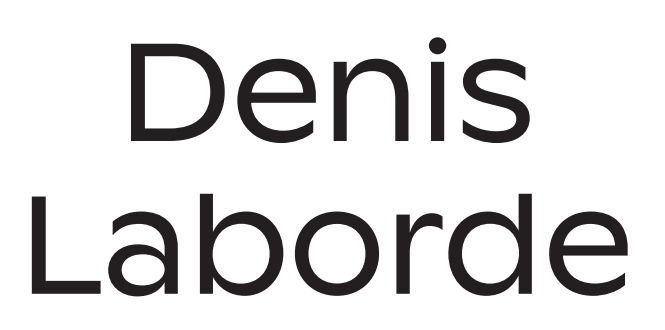


Ancien directeur scientifique de l'Institut de recherche stratégique de l'École militaire (Irsem, ministère des Armées), Frédéric Ramel est aujourd'hui membre du Centre de recherches internationales (Ceri) et directeur du département de science politique de Sciences Po. Ses recherches portent sur les études stratégiques et sur les liens entre relations internationales et pensée politique. II est l'un des pionniers de l'étude des usages de l'esthétique (et notamment de la musique) dans les relations internationales, à travers l'ouvrage qu'il codirige avec Cécile Prévost-Thomas en 2018 : International Relations, Music and Diplomacy. Sounds and Voices on the International Stage 1.

\begin{abstract}
Denis Laborde: Frédéric Ramel, vous êtes un spécialiste internationalement reconnu de l'analyse des relations internationales. Vous dirigez le département de science politique de Sciences Po. Que vient donc faire la musique dans vos travaux?
\end{abstract}

Frédéric Ramel : Au-delà d'une formation musicale jusqu'à mon entrée à l'université et donc d'un intérêt personnel pour cet art, il faut reconnaître que les liens entre musique et politique sont nombreux. Par exemple, ne dit-on pas que «la musique adoucit les mœurs»? Cette formule, très ancrée dans notre sens commun, a une histoire. Nous la tenons des Grecs, lesquels font de la pratique musicale une activité nécessaire à l'éducation du citoyen. Au livre VIII (1339a) de ses Politiques, Aristote soutient que «la musique fait tendre à la vertu dans la mesure où $[. .$.$] tout comme la gymnas-$ tique procure une certaine qualité au corps, la musique donne au caractère une certaine qualité en habituant ses auditeurs à être capables de jouir de plaisirs droits, ou qu'elle contribue à une existence sage » (Aristote 1990: 528-529). Activité nécessaire sur le plan éducatif, mais activité également modératrice au sein de cités tournées vers la guerre. En effet, la musique adoucit les mœurs car elle assagit les tendances parfois belliqueuses des citoyens. C'est le point de vue aussi de Montesquieu: «Il faut donc regarder les Grecs comme une société d'athlètes et de combattants. Or, ces exercices, si propres à faire des gens durs et sauvages, avaient besoin d'être tempérés par d'autres qui pussent adoucir les mœurs. La musique, qui tient à l'esprit par les orǵanes du corps, était très propre à cela.» (Montesquieu 2011 [1748], 1. IV : 8²)

Le pouvoir d'adoucir est ainsi cantonné aux affaires du dedans, celles du dehors étant présidées par la guerre.

\section{L.: La musique ne serait donc qu'affaire de for intérieur?}

F. R. : Il faut bien dire que les relations internationales modernes ne semblent pas avoir modifié cette perspective, dans le sens où c'est la recherche de la survie qui caractérise d'abord et avant tout les interactions entre États, selon le modèle politique classique hérité des traités de Westphalie signés en 1648. La musique et les musiciens demeurent alors des ressources en vue de renforcer l'énergiie combative au front. Certes, les chefs de gouvernement procèdent quelquefois à des commandes musicales destinées à célébrer la paix. Mais ces moments se révèlent fort épars et ne visent pas à adoucir les mœurs dans le temps long. En effet, en raison de son caractère anarchique (absence d'une autorité supérieure qui fixe les normes et contrôle les conduites), le système politique international adopte une attitude suspicieuse à l'égard des dynamiques propres aux formes d'intervention qu'il ne contrôle pas. C'est compter sans l'engagement de certains musiciens eux-mêmes, qui, à travers leur détermination, ont pour ambition de promouvoir des formules alternatives à l'âpreté du monde contemporain.

\section{LA DIPLOMATIE}

DES CÉLÉBRITÉS SELON BONO

D. L.: Selon vous, trois figures de musicien se détachent par leur manière de tisser des liens étroits entre art musical et diplomatie au long de leur parcours. Vous suggérez que nous nous arrêtions successivement sur les cas de Bono, de Daniel Barenboïm et enfin du violoncelliste Yo-Yo Ma. Chacun d'eux cultive, dites-vous, «une certaine façon d'adoucir les relations internationales». N'est-ce pas un peu présomptueux?

F. R.: La portée est certes limitée, mais le geste est riche d'enseignements, qu'il s'agisse du répertoire d'action mobilisé en matière diplomatique ou bien tout simplement de la place du musicien dans la cité. Commençons par Bono, puisque vous m'y invitez. Son action s'inscrit dans un contexte très particulier: la campagne «Jubilée 2000» menée tout au lonǵ des années 1990 pour œuvrer à l'annulation de la dette des pays pauvres très endettés. Bono s'engage dans cette campaǵne avec beaucoup d'enthousiasme et il met son aura de leader du groupe irlandais U2 au service de cette cause. Son engagement ne cessera de gagner en intensité, au point qu'il créera en 2002 sa propre organisation non gouvernementale: DATA (Debts, Aids, Trade, Africa), laquelle fusionnera avec One Campaiǵn pour devenir en 2007 l'orǵanisation ONE, d'après le titre d'un des tubes du groupe U2 (1991). Cette structure entend lutter contre l'extrême pauvreté et les maladies évitables, en particulier en Afrique. L'implication de Bono illustre bien le phénomène qualifié par Andrew Cooper (2008) de «diplomatie des célébrités ». 


\section{Entretien}

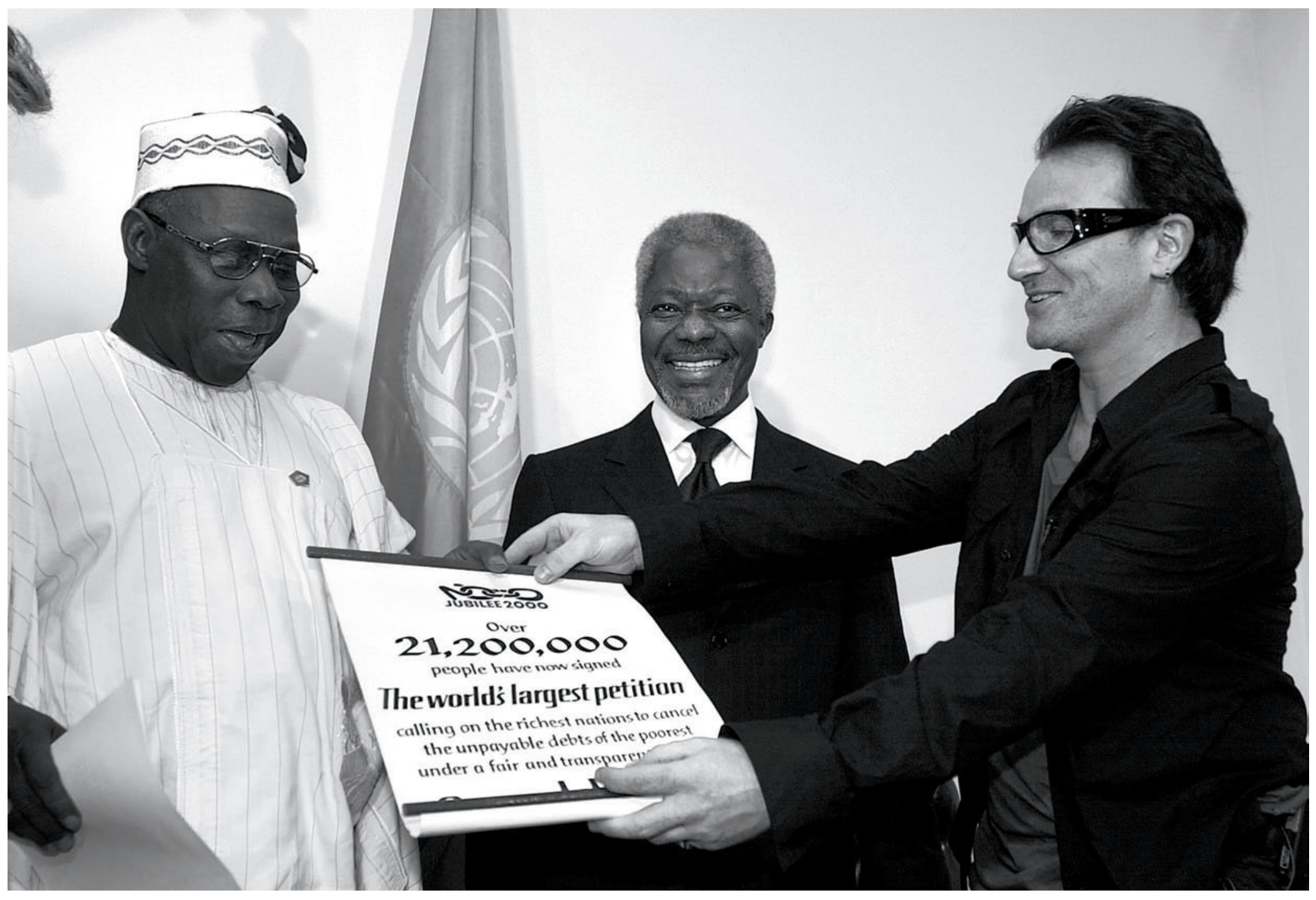

Le Président nigérian Olusegun Obasanjo (à gauche), le secrétaire général des Nations Unies Kofi Annan (au centre), et Bono, du groupe rock U2, montrant une pétition signée par 21 millions de personnes adressée aux dirigeants mondiaux du G8,

pour les presser de supprimer la dette des pays pauvres durant le sommet des Nations Unies du 7 septembre 2000. Photo de Chris Hondros/Newsmakers/Getty images. 
D. L.: Par quels processus ce type d'actions, qui contourne les canaux de la diplomatie d'État pour miser sur l'opinion publique, peut-il remplir une fonction diplomatique?

F. R.: Ce type de diplomatie joue sur plusieurs leviers: l'affinité élective entre la défense d'une cause et le monde du spectacle au sein duquel les émotions d'un artiste et son engagement contribuent à fabriquer son imaǵe; le développement des technologies de l'information et de la communication qui permet une diffusion à la fois rapide et véloce des prises de position adoptées par les artistes; le décentrement de la scène diplomatique qui n'est plus obligatoirement monopolisée par les représentants des États.

\section{L.: Comment caractérisez- vous cette stratégie qui relève de la diplomatie des célébrités?}

F. R.: C'est une forme diplomatique dont le répertoire d'actions présente quatre particularités. Premièrement, la célébrité diffère de l'élite politique car elle ne tire pas son aura d'une élection ou de l'exercice de responsabilités politiques. En d'autres termes, elle se définit comme autonome par rapport à la sphère publique. Deuxièmement, elle privilégie des moyens de communication de masse au profit d'une cause, ce que l'on pourrait désigner par la diplomatie du mégaphone. Troisièmement, la diplomatie des célébrités se veut en articulation avec la diplomatie officielle: "Les individus ne doivent pas seulement posséder d'amples compétences de communication, un sens de la mission [...]. Ils doivent entrer dans le monde diplomatique officiel et opérer dans les relations complexes avec les officiels de l'État. » (Cooper 2008: 7) Quatrièmement, la célébrité doit devenir un interlocuteur reconnu par les diplomates officiels.

\section{L.: Alors revenons à Bono,} si vous le voulez bien, et à cette idée d'une diplomatie qui s'exercerait «de biais», en tirant son origine à l'extérieur du cadre formel de son exercice politique pour y retourner ensuite. De ce point de vue, comment interpréter le fait que Bono a été en lice pour le prix Nobel à trois reprises en 2003, 2005 et 2006 et qu'il a reçu le titre d'«Homme de la paix» en 2008 des mains de Bertrand Delanoë, maire de Paris?
F. R.: Dans le cas de Bono et de son action en faveur d'une annulation de la dette des pays pauvres et surendettés, mais aussi, d'une façon plus générale, pour une présence de l'Afrique au cœur des préoccupations relatives au développement, il faut noter deux choses. La première, c'est que les années 2000 correspondent à une surexposition médiatique de Bono, avec le film The Million Dollar Hotel, sa participation à l'album Shine... La seconde, c'est que les modalités de l'action diplomatique qu'il engage à ce moment, car c'est vraiment de cela qu'il s'agit, se nourrissent de rencontres directes avec les chefs d'État occidentaux du G8. Il parle avec Georǵe Bush Jr, Tony Blair, Gerhard Schröder et Paul Martin au sommet du G8 de Gleneaǵles en 2005, puis lors de celui de 2007. Il a aussi des entrevues en 2005 avec Horst Köhler, ancien président allemand, alors président du FMI, ainsi qu'avec Lula en 2005, puis Medvedev en 2010, sans oublier le président du Nigeria Oluseǵun Obasanjo. Il participe au Forum économique mondial de Davos régulièrement depuis 2001. Bono mène donc une activité très intense basée sur le face-à-face. C'est en ce sens que l'on peut parler de diplomatie.

\section{L.: Mais il prend également la parole dans des arènes dont la visibilité et l'audience sont publiques.}

F. R.: Bien entendu, et j'irais même plus loin. Cette implication diplomatique et les prises de parole auxquelles elle donne lieu se nourrissent de croyances religieuses. Bono n'hésite pas à mettre en avant la foi comme moteur de son engagement et à insister sur le fait que les valeurs qu'elle prône sont fondées sur la tolérance, qui lui fut inculquée dès son plus jeune âge par sa mère protestante et son père catholique. La pluralité d'identités dont témoigne sa trajectoire biographique offre également un socle sur lequel Bono s'appuie pour justifier son action: en tant qu'Irlandais, il incarne un des représentants de la musique anglaise avec $\mathrm{U} 2$, mais aussi un artiste profondément postcolonial à travers son implication pour l'Afrique.

Dès lors, la proximité avec les leaders politiques s'établit aussi grâce à la musique. Ceux qui ont eu une pratique instrumentale ou jouent encore d'un instrument et qui apprécient le mouvement pop se révèlent particulièrement ouverts aux revendications de Bono: Clinton et le saxophone, Blair et la guitare, ou encore l'ancien président de la Banque mondiale James Wolfensohn et le piano. En déployant cette «diplomatie des célébrités», Bono n'ignore pas des modes d'action plus classiques afin de peser sur les décisions des chefs d'État. C'est ainsi qu'il a pu déposer officiellement cette pétition réclamant l'annulation de la dette des pays pauvres très endettés - 21,2 millions de signatures provenant de cent soixante pays -, lors du Sommet du millénaire aux Nations unies en septembre 2000.

Les interventions diplomatiques de Bono entendent influencer directement les responsables politiques. Ici, c'est donc la parole du chanteur dans l'espace public plus que sa voix artistique dans ses chansons qui tente d'adoucir les conditions de vie ordinaire des individus les plus vulnérables, mais aussi les conduites des plus puissants à l'égard de ces derniers. Cette entreprise de lobbying suscite des réactions parfois fort critiques de la part d'autres artistes, lesquels n'y voient qu'une forme de connivence avec les dominants, voire de transgression par rapport aux idéaux dont seraient porteurs les artistes. Bob Geldof ou Mick Jagǵer ne sont pas très tendres à l'égard de Bono. Étant devenu un des leurs, celui-ci ne ferait qu'accepter des compromis, pour ne pas dire des compromissions, avec de vulgaires politiciens (Cooper 2008).

\section{LA DIPLOMATIE À VOIES MULTIPLES SELON BARENBOÏM}

\section{L.: Si le cas de Bono et du groupe U2 est bien connu du grand public, du côté des institutions liées à la musique savante occidentale, l'engagement de Daniel Barenboïm - et son amitié avec Edward Saïd - est tout à fait emblématique d'une forme de diplomatie assez particulière, que vous caractérisez comme une diplomatie «à voies multiples».}

F. R. : En effet. Depuis plusieurs décennies, le conflit israélo-palestinien constitue une ligne de clivage majeure dans l'histoire du système international post-1945. Dans ce contexte, «adoucir» les relations entre Israéliens et Palestiniens relève sans doute de la gageure, voire de l'utopie. À première vue, les actions entreprises par Daniel Barenboïm ne s'inscrivent pas dans la perspective d'une réconciliation politique. Lui-même, d'ailleurs, ne revendique aucune visée politique stricto sensu : il n'est pas politicien mais musicien (Barenboïm 2013).

Néanmoins, ses diverses initiatives 
musiciennes se présentent comme autant de moyens de lutte contre l'âpreté de ce conflit. Rappelons que Daniel Barenboïm est issu d'une famille juive d'origine ukrainienne et russe, installée en Arǵentine. Il est né à Buenos Aires en 1942 et rejoint Israël, dont il a aussi la nationalité, en 1952.

\section{L.: Peut-être peut-on préciser ici qu'il a aussi un passeport palestinien depuis 2008.}

F. R.: Il est vrai que cela fait partie de ce que l'on peut qualifier de «cas» Barenboïm. Il a d'ailleurs aussi un passeport espagnol, mais cela n'entre pas en liǵne de compte ici. Pianiste prodige, il est l'élève de son propre père. En 1957, il joue le Concerto pour piano $n^{\circ} 1$ de Prokofiev au Carnegie Hall de New York. À l'âge de 15 ans, outre sa carrière de pianiste, il étudie la direction d'orchestre et son talent le conduit à diriǵer les formations orchestrales les plus prestigieuses: l'Orchestre symphonique de Chicago, la Scala de Milan, l'Orchestre de Paris (de 1975 à 1989)... Depuis 1992, il est à la tête de l'Opéra national de Berlin et c'est là qu'il a fondé en 2012 l'Académie Barenboïm-Saïd, dont nous allons parler.

\section{L.: Pour l'anecdote, on peut d'ailleurs souligner que c'est lui qui dirigea le fameux Mauerfallkonzert, le Concert de la chute du mur, le 12 novembre 1989 à la Philharmonie de Berlin, dans un contexte politique singulier.}

F. R.: Ce qui est une autre forme de diplomatie, en effet. Dans les années 1990, Daniel Barenboïm rencontre Edward Saïd, universitaire américano-palestinien qui enseigne alors la littérature comparée à Columbia University (New York). Cette rencontre est le déclencheur d'un projet original: la création d'un orchestre réunissant jeunes musiciens palestiniens, israéliens, ou venus d'autres États arabes de la région (Barenboïm et Saïd 2003). Ce projet se concrétise en 1999 à l'occasion d'un séminaire organisé à Weimar pour célébrer le $250^{\mathrm{e}}$ anniversaire de la naissance de Gœthe (Ramel 2014). Le nom de l'orchestre fait d'ailleurs référence à un poème de Goethe promouvant les relations entre l'Orient et l'Occident ${ }^{3}$. À sa création, l'orchestre reste basé à Weimar pendant deux années. Il bénéficie ensuite du soutien de l'Orchestre symphonique de Chicago (dont Barenboïm est directeur musical à l'époque).
En 2002, le gouvernement d'Andalousie prend le relai de l'Orchestre de Chicago. Basé à Séville, le célèbre West-Eastern Divan Orchestra se réunit dès lors chaque été afin de préparer une tournée de concerts dans les villes du pourtour méditerranéen. Le Divan Orchestra est aujourd'hui installé à Berlin, où il bénéficie depuis 2016 de l'aide administrative, financière et logistique de l'Académie Barenboïm-Saïd, haut lieu de formation des jeunes musiciens, qui dispose d'une salle de concert innovante baptisée «Pierre Boulez», au nom de l'amitié d'une vie qui aura lié le chef d'orchestre israélo-argentin de Berlin au compositeur et chef d'orchestre français de Baden-Baden 4 .

\section{L.: Peut-on dire du Divan Orchestra qu'il est un «orchestre de paix»?}

F. R.: Oui, d'une certaine manière. Car paradoxalement, l'institution qui garantit les conditions du succès de cette aventure humaine a tendance à bloquer l'élan de paix. Cela se joue au niveau de la motivation de ceux qui prennent part à cette aventure. Des travaux scientifiques importants conduits par des ethnologues montrent en effet que les raisons pour lesquelles les jeunes musiciens participent à cet orchestre œcuménique tiennent bien plus à une stratégie personnelle de carrière qu'à une visée altruiste ou au souci de contribuer par l'orchestre symphonique à construire la paix dans le bassin méditerranéen. Ils tendent à privilégier leur propre carrière musicale, s'éloignant de l'esprit qui guida les créateurs de l'ensemble (Beckles Willson 2009). D'autre part, Daniel Barenboïm lui-même tient à séparer, cette fois encore, l'expérience du Divan Orchestra du processus de paix, en ramenant l'orchestre à une expérience artistique avant tout et en indiquant clairement: «Le Divan n'est pas une histoire d'amour, ni une histoire de paix.» (Barenboïm, in Vulliamy 2008) Mais au-delà de ces deux interprétations qui semblent éloigner le rôle de l'orchestre de notre réflexion, en le transformant en «non-sujet» pour les relations internationales, il convient de souligner l'ambition majeure qui préside à ce projet: transformer les représentations de l'autre, former un nouvel imaginaire grâce à l'outil symphonique.
D. L.: Analysons le fait que Daniel Barenboïm met en avant l'exigence artistique comme une tentative de neutraliser la part émotionnelle de toute construction diplomatique,

cette part livrée aux «passions identitaires» qui s'expriment dans le cadre géopolitique israélo-palestinien saturé de mobilisations émotionnelles. De ce point de vue, le Divan Orchestra aurait pour fonction de ramener la question de l'émotion politique au niveau d'une pure praxéologie de la coordination musicienne au sein de l'orchestre symphonique. Ce serait une manière de théoriser I'orchestre, comme Albert Hirschman théorise le lien entre passions et intérêts au sein des échanges économiques: au prix d'un léger déplacement (culturel), il deviendrait possible de transformer des passions dont la confrontation paraît insoluble en une action coordonnée (l'œuvre symphonique), à prévisibilité prescrite (le concert), qui vise au bien commun (une émotion artistique partagée).

F. R.: On peut effectivement analyser cette économie symbolique en ces termes, me semble-t-il. D'ailleurs, on dit souvent, pour parler de l'orchestre: «la république souveraine et indépendante du West-Eastern Divan» (Barenboïm 2008). Cette expression de nature politique suggèere l'idée d'une intégration entre les musiciens, créant ainsi un «tout organique»-idée que Barenboïm reprend au chef d'orchestre Wilhelm Furtwänǵler ${ }^{5}$-, et en appelle à un imaǵinaire qui se situe aux antipodes d'un conflit de plus en plus enkysté, dont l'issue s'éloigne vers un imperceptible horizon. Rappelons-le - bien que cette idée soit elle-même ambiguë (Jung et Ramel 2018) -, Barenboïm affirme qu'il ne souhaite en aucun cas exercer un rôle politique, ni même influencer les leaders politiques. Son répertoire d'actions diffère radicalement de la «diplomatie des célébrités » cultivée par Bono. Néanmoins, en disséminant une autre représentation des ennemis à travers l'expérience musicale, il bouscule les perceptions premières, quitte à susciter en retour de violentes réactions, y compris de la part du gouvernement israélien.
D. L.: À I'instar de la polémique de grande envergure qu'il a suscitée à Jérusalem, le 7 juillet 2001, en dirigeant des extraits de l'opéra de Richard Wagner Tristan et Iseult, alors qu'il était à la tête de l'Orchestre 
Un musicien peut-il adoucir les relations internationales?

Avec Frédéric Ramel et Denis Laborde

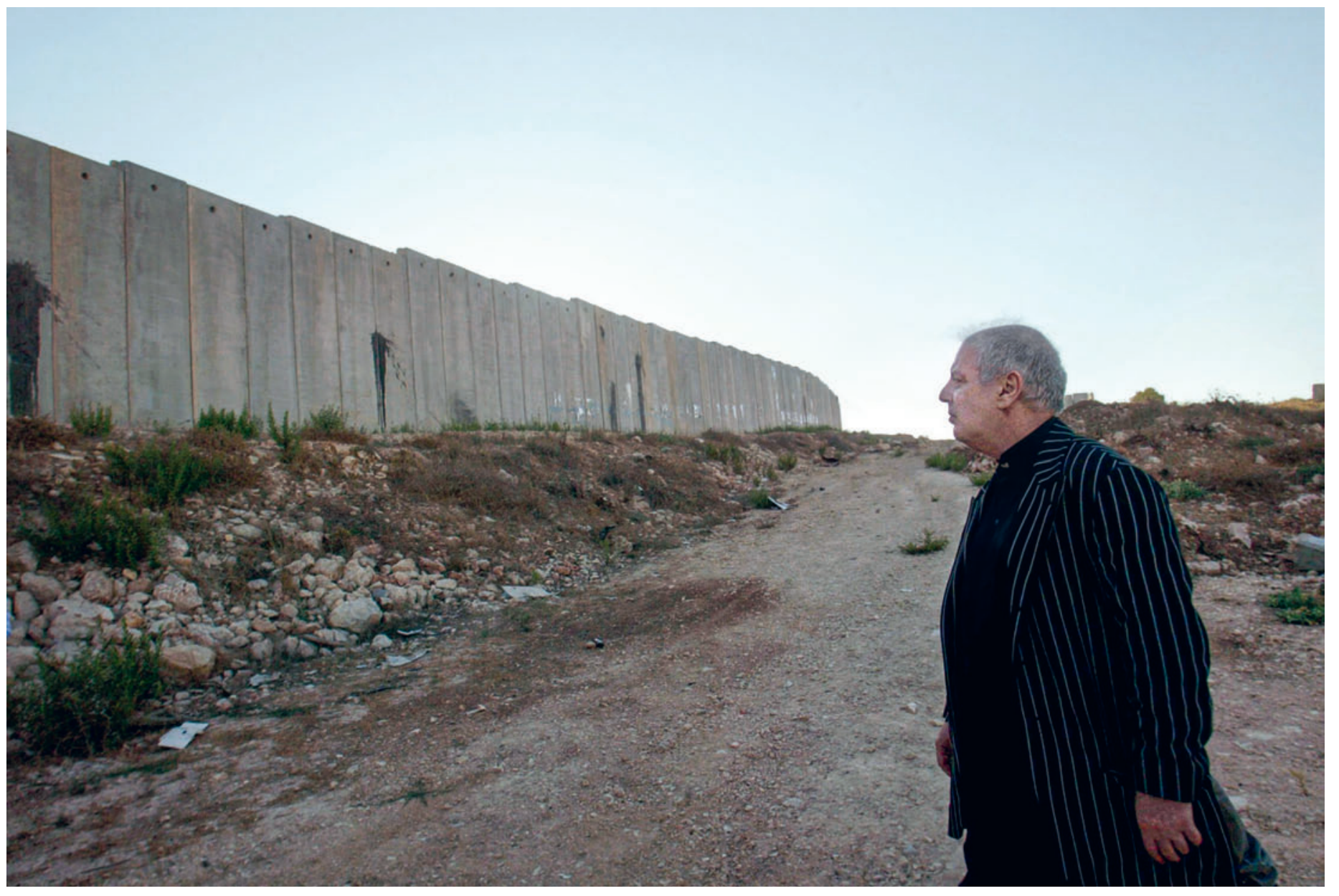

Daniel Barenboïm à Ramallah, 20 août 2005 @ Osama Silwadi/Palestine Image Bank. 


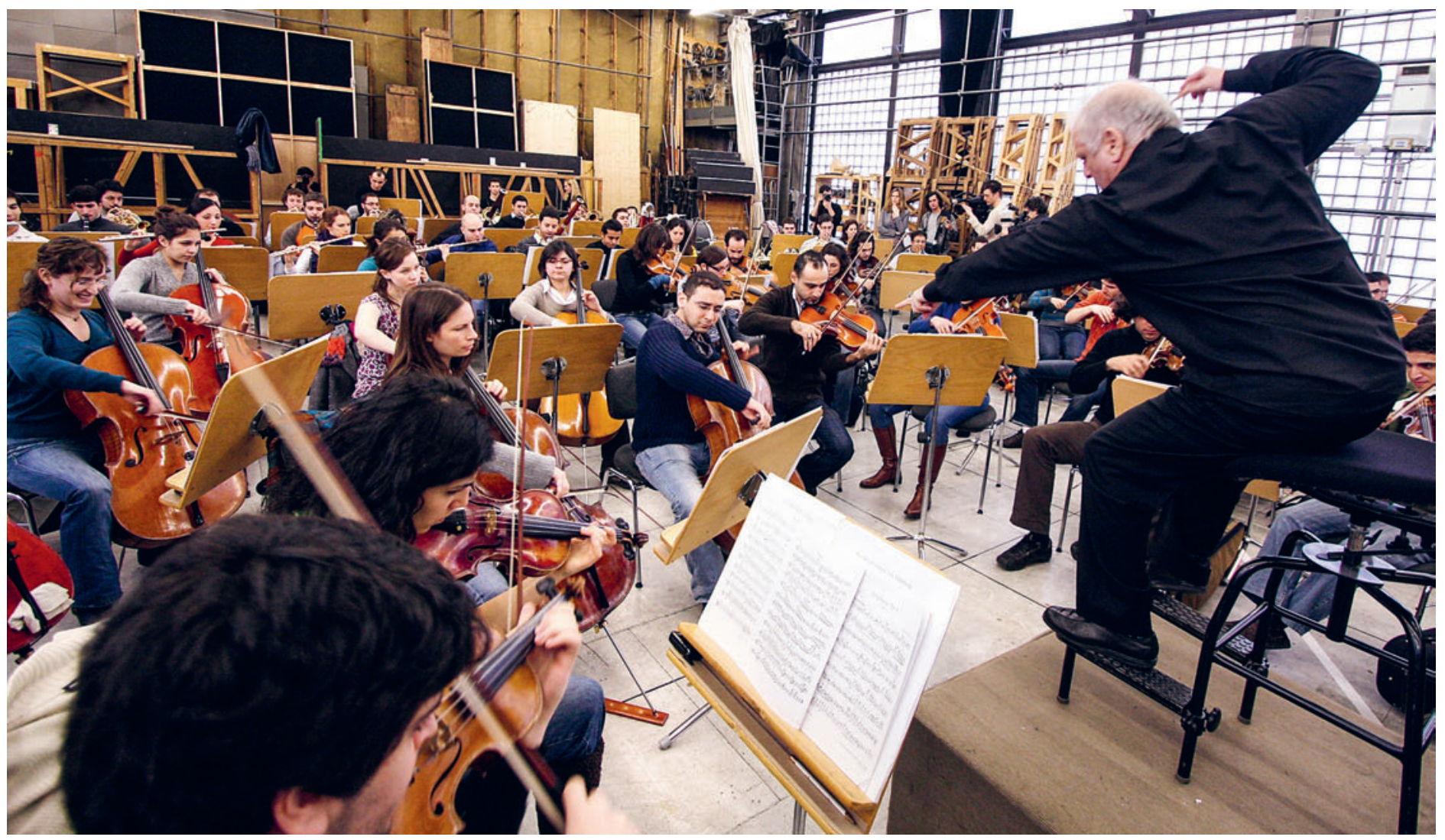

West Eastern Divan Orchestra, concert au Staatsoper unter den Linden, Berlin, première le 12 janvier 2009 c) akg-images / Thomas Bartilla. 
philharmonique de Berlin (Laborde 2002). Le concert avait suivi un cours très inhabituel: après un long échange avec le public, il avait invité les spectateurs qui ne souhaitaient pas entendre Tristan et Iseult à quitter la salle. Edward Saïd $a$ analysé ce blasphème dans un article paru dans Le Monde diplomatique avec ce sous-titre: «Peut-on aimer la musique du compositeur préféré de Hitler?» (Saïd 2001). Là encore, Barenboïm brouille les cartes.

F. R.: Oui, Barenboïm le clame haut et fort: la musique avant tout. Pourtant, l'idée selon laquelle un orchestre représente plus qu'un groupe de professionnels jouant ensemble de la musique n'est pas récente. L'histoire des usages du mot montre à quel point l'orchestre a servi de métaphore pour parler du corps politique (Spitzer et Zaslaw 2005). Cela fait partie des topiques de nos représentations culturelles : l'orchestre symphonique incarne une forme de société politique en miniature basée sur la coopération pour atteindre un but commun. Lorsque le 21 septembre 2007, le secrétaire général des Nations unies, Ban Ki-moon, confère à Daniel Barenboïm le statut de «Messager de la paix», ce dernier convoque à nouveau cette conception de l'orchestre comme un tout intégré, pour faire de l'orchestre, plus que de lui-même, le véritable messager de la paix, bien que la formation soit «en exil» (Barenboïm 2018: 19). Puis, le 29 février 2016, c'est au tour du West-Eastern Divan Orchestra lui-même d'être nommé par le secrétaire général des Nations unies à la fonction de «Défenseur mondial des Nations unies pour la compréhension multiculturelle». L'orchestre bénéficie ainsi d'une aura mondialisée: il devient l'outil d'une diplomatie qui vise à administrer la preuve par l'art qu'un autre futur est envisageable.

\section{L.: Mais en quoi ces formes d'action diplomatique qui mettent en jeu la mobilisation iconique de l'orchestre symphonique s'apparentent- elles à une diplomatie «à voies multiples»?}

F. R. : La diplomatie à voies multiples est une catégorie de l'action diplomatique qui a été étudiée dans les années 1990 par Louise Diamond et John McDonald (1991), puis reprise dans une publication majeure dirigée par James Notter et à nouveau
Louise Diamond (1996). C'est ce qu'ils nomment la multi-track diplomacy. Cette diplomatie enrichit la compréhension des négociations internationales en soulignant l'existence de sentiers parallèles aux rencontres formelles ou informelles entre dirigeants politiques. Des organisations non gouvernementales, des structures confessionnelles ou mêmes universitaires peuvent ainsi contribuer à rapprocher les points de vue et transformer les représentations de l'autre en vue de créer les conditions favorables à la résolution d'un conflit armé. La diplomatie à voies multiples diversifie les canaux de communication entre les différentes parties en cultivant une double finalité : humaniser l'ennemi en le singularisant; influencer la voie officielle en visant les racines historiques et culturelles d'un conflit. En insistant sur la nécessité de changer le regard porté sur l'ennemi à l'échelle interindividuelle, Daniel Barenboïm fait du Divan Orchestra l'outil d'une diplomatie à voies multiples. D'ailleurs, le documentaire réalisé sur l'orchestre par Paul Smaczny, qui reçut un Emmy Award en 2005, s'intitule: Knowledge is the Beginning. Qui plus est, la mise en place de la Fondation BarenboïmSaïd à Berlin en 2016 offre un nouvel espace de formation instrumentale de haut niveau, mais aussi d'éducation pour des musiciens qui intègrent la visée d'une transformation du rapport à l'autre dans leur propre engagement artistique. Un tel espace entend "promouvoir un développement humain qui culmine dans la compréhension mutuelle» (Barenboïm 2018: 110, traduction de F. R.). Si l'œuvre musicale est bien la finalité de l'action collective, son processus de production devient un outil qui doit permettre de reconfigurer les liens entre Israéliens et Palestiniens.

\section{LA DIPLOMATIE TRANSPROFESSIONNELLE SELON YO-YO MA}

D. L.: Si Daniel Barenboïm
concentre ses initiatives
musiciennes sur la relation
de face à face entre Israéliens
et Palestiniens, d'autres
musiciens bâtissent des projets
à vocation mondialisée
dans lesquels l'orchestre
symphonique ne joue
pas nécessairement un rôle
de premier plan. C'est le cas
du violoncelliste Yo-Yo Ma.

F. R. : Yo-Yo Ma est également un musicien prodige qui gagne rapidement une reconnaissance internationale. Né à Paris en 1955 de parents chinois, tous deux musiciens, il a étudié au conservatoire de Saint-Germainen-Laye, puis sa famille ayant déménagé à New York, il y poursuit sa formation à la Juilliard School. En 1998, Yo-Yo Ma crée le Silk Road Project. Son idée est de contrer, par la musique, le processus d'uniformisation engendré par la mondialisation en mettant en avant ce que celle-ci a de positif: la possibilité pour des musiciens du monde entier de travailler ensemble. Il fait alors de l'histoire de la route de la soie un modèle de collaboration culturelle porteur d'innovation et il propose à des musiciens issus des pays traversés par cet ancien réseau de routes commerciales d'œuvrer avec lui à la création d'une attitude artistique fondée sur la mise en commun des différences.

\section{D.L.: Ajoutons ici que Yo-Yo Ma fait partie de ces musiciens qui prêtent un intérêt particulier à l'anthropologie, au point de suivre un cursus d'anthropologie à I'université de Harvard, où il a obtenu sa licence en 1976. En tout cas, son Silk Road Project se déroule à une échelle mondiale, avec des concerts, des ateliers de formation, des résidences d'artistes, le tournage de films documentaires, dont l'opus magnum The Music of Strangers: Yo-Yo Ma \& The Silk Road Ensemble, réalisé par Morgan Neville en $2016^{6}$... Et le siège de cette «Route de la soie» est à Boston.}

F. R.: Oui, Yo-Yo Ma vit aujourd'hui à Boston. Il collabore avec les universités locales et avec l'orchestre symphonique, il est aussi engagé dans de nombreux projets avec l'Orchestre symphonique de Chicago. Il bénéficie donc sur place d'appuis qui lui permettent d'installer ses outils de travail. Le Silk Road Project se distingue des projets précédemment évoqués par son objectif de favoriser la collaboration artistique multiculturelle. L'ensemble instrumental va done façonner un répertoire constitué de toutes les sortes d'héritages de musiques de tradition orale et d'improvisations en tout genre. Au fil des années, le Silk Road Project s'est enrichi de programmes complémentaires en matière éducative: Silk Road Encounter, pour travailler à l'élaboration d'outils pédagogiques; Silk Road Connect, pour structurer des interventions au sein 
d'écoles aux États-Unis; The Arts and PassionDriven Learning Institute, un institut universitaire créé en 2012 et co-animé avec le Harvard Graduate School of Education. Morgan Neville a bien reflété l'esprit qui anime ces différentes activités dans le documentaire que vous citez.

\section{L.: Comment isole-t-on un item «diplomatie» dans cette prolifération d'actions à ce point diversifiées?}

F. R. : Contrairement aux interventions de Bono ou même de Daniel Barenboïm, qui se situent au cœur d'enjeux politiques internationaux identifiés (le développement et le conflit israélo-palestinien), celles de Yo-Yo Ma ne se construisent pas autour d'une «revendication pivot». Elles relèvent de l'action culturelle transnationale: organiser des concerts, proposer des formations, assurer des animations scolaires. Elles se déploient sur un autre registre, celui de la «transprofessionnalisation de la diplomatie». Celle-ci se définit comme «un développement productif qui reflète l'espace diplomatique élargi et l'intensification des interconnexions et des réseaux mondiaux, ainsi que les nouvelles possibilités [que de telles actions] offrent pour la pratique de la diplomatie dans différents milieux » (Constantinou et al. 2016: 3 ; traduction de F. R.) : ce mouvement de transprofessionnalisation révèle tout d'abord une dynamique opérant au sein même de la société civile. Il doit son apparition et son efficience à l'essor d'acteurs culturels qui, dans leur propre métier et de par leur carrière internationale, cultivent des relations diplomatiques. Alors, bien sûr, les tenants d'une conception classique de la diplomatie en tant qu'activité monopolisée par les États et objet d'une spécialisation fonctionnelle sont particulièrement rétifs à une telle extension du terme «diplomatie» vers d'autres secteurs de l'activité des sociétés humaines (Kennan 1997). Il n'en demeure pas moins qu'une «diplomatisation de la vie sociale» (Neumann 2012) est bien à l'œuvre. Elle témoignne d'une pratique qui vise à reconnaître et à enǵager une relation avec d'autres en vue de mener une action commune ou d'élaborer de nouvelles normes (trans)culturelles. C'est exactement ce à quoi se consacre Yo-Yo Ma à travers son action musicale, notamment auprès des jeunesses afro-américaines, en partenariat avec l'Orchestre symphonique de Chicago (National Gallery of Art 2015). Le propos de Yo-Yo Ma est assez limpide: il s'aǵit de fabriquer un langage commun afin de transcender des traditions musicales différentes, sans les gommer. C'est une manière d'installer la diplomatie au cœur même du Silk Road Project. D'une part, les instrumentistes invités proposent des œuvres issues de leurs propres traditions musicales; d'autre part, ils participent à l'invention de nouvelles pièces construites ensemble dans l'idée d'élaborer un nouveau répertoire. Les répétitions deviennent ainsi des laboratoires d'improvisation donnant vie à des œuvres qui traversent les frontières, à l'instar de l'Indien Sandeep Das, joueur de tabla, ou de l'Iranien Kayhan Kalhor, pratiquant le kamancheh (Segran 2013).

\section{L.: Mais ici se pose quand même la question de la circulation des artistes.}

\section{F. R. : Effectivement, nous sommes} là au plus près des modalités classiques de la relation diplomatique. Les acteurs sociétaux qui élaborent des projets à la façon du Silk Road Project sont associés aux chancelleries. D'une part, les chancelleries facilitent la circulation des musiciens, mais réciproquement, en collaborant avec des artistes, elles enrichissent leur propre répertoire d'actions dans l'environnement international contemporain. Cette particularité donne corps à une seconde dimension de la transprofessionnalisation de la diplomatie.

À la suite du déclenchement de la guerre contre le régime des talibans en Afǵhanistan, le Département d'État américain soutient l'organisation du Smithonian Folklife Festival sur le Mall de Washington. La visée de ce festival est de «découvrir les traditions vivantes du monde entier» en travaillant avec les communautés. Mais rien n'est plus proche des représentations diplomatiques classiques que d'installer le festival sur le Mall de Washington. En 2002, donc, un partenariat se construit avec la Fondation Agan Khan et le festival propose aux musiciens du Silk Road Project d'organiser un concert à cet endroit. Ce concert illustre l'articulation étroite entre diplomatie culturelle (menée par les États), action culturelle (réalisée par des acteurs sociétaux) et diplomatie publique (en vue de transformer l'image des États tant dans l'espace national qu'à l'étranger). Par ailleurs, les représentations que souhaite disséminer l'administration Bush dans le contexte post-11-Septembre se trouvent ici mises en avant par le festival lui-même: développement de soi, rencontre de l'altérité, ouverture aux autres, découverte d'autres cultures, tels sont quelques-uns des arguments qui accompaǵnent le déroulé de cette manifestation (Lanǵenkamp 2014).

\begin{abstract}
D. L.: Pourtant, il me semble que le Silk Road Project ne s'inscrit dans ce lien avec les chancelleries que d'une manière tangentielle. Jouer dans les antres du pouvoir n'est pas au cœur de son propos, contrairement à ce que vous nous avez expliqué, par exemple, du projet de Bono, qui vise ouvertement à mobiliser frontalement la représentation politique.
\end{abstract}

F. R. : Je dirais que les formes diplomatiques que cultivent les musiciens du Silk Road Project révèlent surtout deux principales tendances à l'adoucissement. La première réside dans le rapport au passé. Plusieurs instrumentistes du Silk Road Project proposent des performances de musique traditionnelle dans des musées archéologiques, par exemple. Par là, ils se font les messaǵers du temps, ce qui renvoie bien à la fonction même du diplomate en tant qu'émissaire ayant pour charge de rapprocher les hommes, de rapprocher des entités politiques ou, à la façon d'Hermès, de rapprocher les dieux des hommes (Raymond 2015). Installer la musique des traditions du monde dans les vestiges archéologiques conservés dans les musées permet un rapport direct, sensoriel, aux productions culturelles antérieures.

\section{L.: Une mesure de l'épaisseur du temps?}

F. R. : En tout cas, dans cette perspective, les musiciens du Silk Road Project rendent le passé plus proche de l'auditeur. Mais la seconde forme d'action ouvre pour sa part de nouveaux horizons dans des contextes difficiles. Par exemple, la participation des musiciens du Silk Road Project au Stanford Program on International and Cross-Cultural Education (Programme de Stanford pour l'éducation internationale et interculturelle, SPICE) est très représentative in situ ${ }^{7}$. Un dialogue entre Yo-Yo Ma et le clarinettiste syrien Kinan Azmeh témoigne de la manière dont des instrumentistes conçoivent leur rôle par rapport aux conflits qui les touchent directement, comme la guerre en Syrie (Azmeh et Ma 2016). D'une manière prudente mais engagée, les deux musiciens font part des limites de la musique - et des formes de diplomatie qu'il est possible de lui associer - comme mode de transformation du monde. Azmeh aime à rappeler que l'art n'offre pas de substances médicamenteuses. Pourtant, composer et organiser des performances musicales sont des moyens de répondre 
Un musicien peut-il adoucir les relations internationales? Avec Frédéric Ramel et Denis Laborde

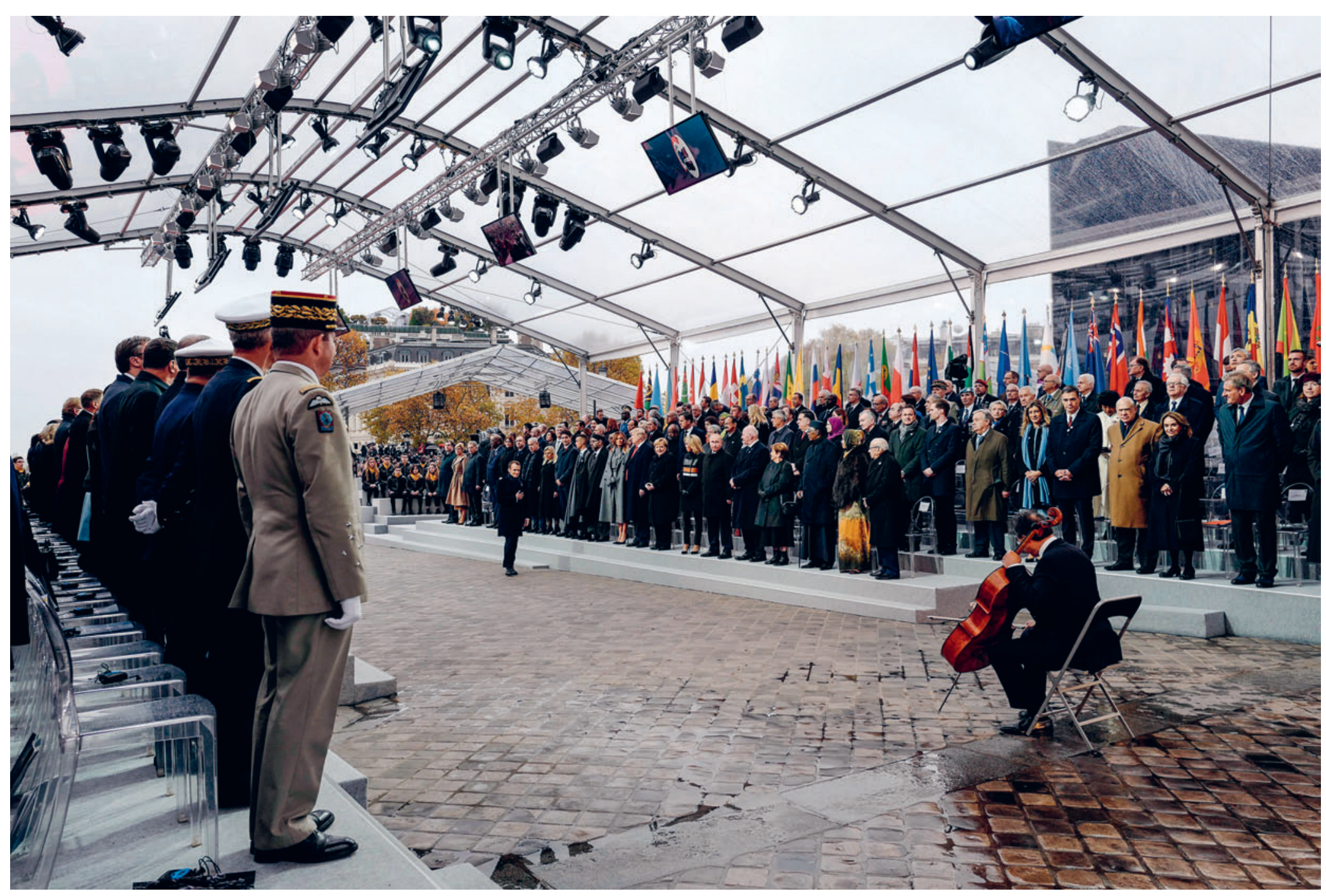

Cérémonie pour le centenaire de l'Armistice de 1918, concert de Yo-Yo Ma, Arc de Triomphe, Paris, 11 novembre 2018 (c) Photo Xavier Popy-Pool/SIPA. 
d'une certaine façon à la situation.

Tous deux considèrent l'action musicienne comme la fabrication de "petites pièces d'un puzzle». Les moments musicaux fabriqués ainsi invitent les auditeurs à demeurer vigílants, à «garder les fenêtres ouvertes »... Ce dialogue entre les deux musiciens est si important pour les tenants du Silk Road Project qu'il est proposé au titre de matériel pédaǵogique pour des formations universitaires consacrées à la place de la musique en situation de crise humanitaire.

\section{L.: C'est à l'aune de cette expérience que vous interprétez le rôle que le protocole a fait jouer à Yo-Yo Ma lors de sa venue à Paris, sous l'Arc de triomphe en 2018?}

F. R.: Exactement. Le 11 novembre 2018, la République française a tenu à commémorer le centenaire de l'Armistice de la Première Guerre mondiale. Dans ce cadre, le président Macron rappelle les dix-huit millions de morts de ce conflit et exhorte «au combat pour la paix». La cérémonie a lieu sous l'Are de triomphe, devant la tombe du soldat inconnu. Devant soixante-dix chefs d'État et de gouvernement, Yo-Yo Ma joue seul au violoncelle la Sarabande de la Suite $n^{\circ} 5$ de Bach. Mais en marge de cette prestation, il fut invité à témoigner de son expérience lors d'une master class programmée dans le cadre du Forum de Paris sur la paix. C'est une manière de célébrer le lien entre musique et diplomatie. D'ailleurs, Yo-Yo Ma est, lui aussi, l'un des messagers de la paix de l'ONU depuis 2006 et il s'est produit à la tribune de l'Assemblée générale des Nations unies lors de la journée de l'organisation le 24 octobre 2008 avec le Silk Road Project.

\section{EN GONCLUSION}

D. L.: En définitive, nous avons distingué trois formes d'action diplomatique, et à chacune d'elles nous avons associé un musicien emblématique: Bono à la diplomatie des célébrités, Daniel Barenboïm à la diplomatie à voies multiples, Yo-Yo Ma à la diplomatie transprofessionnelle. Pourtant, aucun de ces musiciens n'a fait de carrière politique. Et au fond, je m'interroge: cet «idéal du musicien », sa proposition d'un monde irénique n'est-elle pas rendue possible à la seule condition que chacun, sur l'échiquier postcolonialiste, vienne occuper la place prévue pour lui par les puissances dominantes?

F. R.: Effectivement, aucun des musiciens dont il a été question ici n'a exercé de responsabilités politiques. Ils n'entrent donc pas dans la catégorie des professionnels de la politique ayant pour but de peser directement sur les enjeux stratégiques du monde contemporain par l'exercice d'un mandat. C'est d'autre chose qu'il s'aǵit. Néanmoins, s'ils n'embrassent pas une «vocation politique» au sens wébérien du terme, leurs actions témoignent bel et bien d'une pluralisation des registres d'action de la diplomatie (Cornago 2013). Nul ne peut les iǵnorer: par leur notoriété, ils pèsent sur les affaires politico-diplomatiques du monde. Et de ce point de vue, le fait qu'ils cherchent à apaiser les relations intergoouvernementales, à renforcer les modalités de dialogues constructifs, à proposer un nouvel imaginaire commun dans des configurations de conflits armés, ou encore qu'ils cherchent à prodiguer de nouvelles formes sonores et des supports éducatifs fondés sur des expérimentations musicales interculturelles, joue un rôle dans les reconfigurations des représentations de l'altérité qu'ils appellent de leurs vœux. Enfin, le fait qu'ils se produisent dans des lieux symboliques du pouvoir mondialisé (Assemblée générale de l'ONU, Mall de Washington, Arc de triomphe) procure à leurs actions une aura elle-même mondiale. En illustrant la tendance au polylatéralisme qui caractérise les scènes diplomatiques actuelles (Wiseman 2010), ces formes tangentielles de la diplomatie révèlent l'implication des musiciens au cour de la cité, quand bien même les voix qu'ils font entendre ne bénéficient pas de résonances aussi robustes que celles des pouvoirs publics. 
1. Frédéric Ramel et Cécile Prévost-Thomas (dir.), International Relations, Music and Diplomacy: Sounds and Voices on the International Stage, Cham (Suisse), Palgrave Macmillan, 2018

2. Dans la querelle qui oppose les Anciens et les Modernes, Montesquieu ne propose pas de renouer avec ces pratiques antiques, puisqu'il cherche à mieux saisir la spécificité et la supériorité des régimes modernes.
Néanmoins, une aristocratie moderne pourrait contribuer au bon fonctionnement d'une monarchie modérée grâce à la reconnaissance du rôle de la musique dans la formation de l'esprit (Spector 2017).

3. Le Divan occidental-oriental (West-östlicher Divan) est l'un des tout derniers recueils poétiques de Goethe (1749-1832). Les douze livres qui le composent furent publiés entre 1819 et 1827.
Le recueil s'inspire de la poésie soufie de Hāfiz, poète persan du xive siècle, dont Joseph von Hammer-Purgstall (1774-1856) publie une traduction allemande en 1814. Chacun des livres comprend un titre en allemand et un titre en langue arabe. En nommant leur formation West-Eastern Divan Orchestra Daniel Barenboïm et Edward Saïd décident de l'inscrire dans cette histoire des transferts culturels.
4. Ce choix en faveur de Berlin résulte également des fonctions de chef qu'assure Daniel Barenboïm à la Staatskapelle de Berlin.

5. «Furtwängler était convaincu que tout est lié: la musique comme un tout organique. Pour Furtwängler il n'y avait pas de phénomènes indépendants les uns des autres», explique Barenboïm (2004).
6. Voir le site de ce projet: https:// www.silkroad.org/about, ains que le film The Music of Strangers: Yo-Yo Ma \& The Silk Road Ensemble, réalisé par Morgan Neville (États-Unis, 2016,1 h 36 min).

7. Voir le site du programme de I'université de Stanford: https:// spice.fsi.stanford.edu/

\section{Bibliographie}

\section{Aristote}

1990 Les Politiques. Paris, Garnier Flammarion.

Azmeh, Kinan et Ma, Yo-Yo 2016 «Art in a Time of Crisis», SPICE [en lióne], disponible sur: https://fsi-lives.3.us-west-1. amazonaws.com/s3fs-public/ arterisis_silkroad.pdf

\section{Barenboïm, Daniel}

2004 «Warum uns Wilhelm Furtwängler bis heute bewegt », Der Tagesspiegel, 30 novembre; repris en anǵlais: "Why Wilhem Furtwänóler Still Moves Us

Today» (trad. par Brian Currid) [en ligne], disponible sur : https://daniel barenboim.com/ why- wilhelm-furtwangoler-stillmoves-us-today/

(consulté le 12 février 2020).

2008 «Equal before Beethoven», The Guardian, 13 décembre.

2013 «A Musical Path to Peace Talk to Al Jazeera », Al Jazeera, 2 février.

2018 "A school of Acceptance: A Musical-political Idea Becomes a Reality », in Daniel Barenboïm et Michael Naumann (dir.),

The Sound of Utopia: From the West-Eastern Divan Orchestra to the Barenboim-Said Akademie, catalogue d'exposition (Berlin, Barenboim-Said Akademie, 2016), trad. de l'anǵlais par Darrelll Wilkins. Leipziǵ par Darrell Wilkins.

\section{Barenboïm, Daniel} et Saïd, Edward

2003 Parallèles et paradoxes: explorations musicales et politiques, trad. de l'anǵlais par Philippe Babo. Paris, Le Serpent à plumes.

\section{Beckles Willson, Rachel}

2009 «Whose Utopia? Perspectives on the West-Eastern Divan Orchestra», Music \& Politics 3 (2) : 1-21.

Constantinou, Costas M., Conrago, Noé, et McConnell Fiona

2016 «Transprofessional Diplomacy », Diplomacy and Foreign Policy 1 (4) : 1-66.

\section{Cooper, Andrew F.}

2008 Celebrity Diplomacy. Boulder, Paradiǵm.

\section{Cornago, Noé}

2013 Plural Diplomacies: Normative Predicaments and Functional Imperatives. Leiden, Brill

\section{Diamond, Louise} et McDonald, John W.

1991 Multi-Track Diplomacy: A Systems Approach to Peace. West Hartford, Kumarian Press.

\section{Jung, Michae}

et Ramel, Frédéric

2018 «The Barenboïm Case How to Link Music and Diplomacy Studies?», Arts and International Affairs 3 (2) 39-67.

\section{Kennan, George}

1997 «Diplomacy without Diplomats? », Foreign Affairs 76: 198-212.

\section{Laborde, Denis}

2002 «The Unbearable Sound: The Strange Career of Musicoclashes» in Bruno Latour et Peter Weibel (dir.), Iconoclash: Beyond the Images War. Cambridge, MIT Press: 253- 280 .

\section{Langenkamp, Ham}

2014 «Conflicting Dreams of Global Harmony in US-PRC Silk road Diplomacy in Rebekah Ahrendt, Mark Ferraguto et Damien Mahiet (dir.), Musical Diplomacy from Early Modern Era to the Present. Basinǵstoke, Palǵrave Macmillan : 83-100.

\section{Montesquieu}

2011 [1748] L'Esprit des lois, Robert Derathé et Denis de Casabianca (éd.) Paris, Garnier.

\section{National Gallery of Art} et Foundation for Art and Preservation in Embassies (FAPE)

2015 «The Role of Art in Diplomacy: Cultural Citizens table ronde réunissant Yo-Yo Ma, Darren Walker, Theaster Gates et Molly Donovan (modératrice), Washington, 20 avril [en ligne], disponible sur : https://www.youtube.com/ watch?v=2zjO_GhSJ4g (consulté le 08/06/2020)
Neumann, Iver B.

2012 «Globalisation and Diplomacy », in Andrew F. Cooper, Brian Hocking et William Maley (dir.), Global Governance and Diplomacy: Worlds Apart?. Houndmills, Palgrave Macmillan : 15-28.

\section{Notter, James}

et Diamond, Louise

1996 Building Peace and Transforming Conflict:

Multi-Track Diplomacy in

Practice. Washington, IMTD.

\section{Ramel, Frédéric}

2014 "Divan Orchestra: Mutual Middle-Range Transformation », in Brigitte Vassort-Rousset (dir.), Building Sustainable Couples in International Relations: A Strategy Towards Peaceful Cooperation. London, Palǵrave MacMillan : 209-237.

Raymond, Jean-François (de) 2015 L'Esprit de la diplomatie : du particulier à l'universel. Paris, Les Belles Lettres.

\section{Saïd, Edward}

2001《Peut-on admirer la musique du compositeur préféré de Hitler? Barenboïm brise le tabou Waǵner»,

Le Monde Diplomatique 10 : 24-25.

\section{Segran, Elizabeth}

2013 «A Yo-Yo Ma Project Brings Together Musicians From Warring Nations », The Atlantic, 10 octobre.

\section{Spector, Céline}

2017 «Des effets politique de la musique: Montesquieu, les Grecs et l'éducation libérale des Modernes ", Implications philosophiques.org [en liǵne], disponible sur: https://www. implications-philosophiques. org/actualite/une/des-effetspolitiques-de-la-musique-montesquieu-les-grecs-et-leducation-liberale-des-modernes/ (consulté le 08/06/2020)

Spitzer, John et Zaslaw, Neal 2005 The Birth of the Orchestra: History of an Institution, 1650-1815. New York/Oxford, Oxford University Press.

\section{Vulliamy, Ed}

2008 «Bridging the Gap, Part Two », The Guardian, 13 juillet.

\section{Wiseman, Geoffrey}

2010 «Polylateralism. Diplomacy's Third Dimension», Public Diplomacy Magazine 4 : 24-39. 